\title{
Freezing of Spinodal Decomposition by Irreversible Chemical Growth Reactions
}

\author{
Fachbereich Physik, Martin-Luther-Universität, 06099 Halle, Germany \\ Michael Schulz and Benjamin Paul
}

(November 20, 2018)

\begin{abstract}
We present a description of the freezing of spinodal decomposition in systems, which contain simultaneous irreversible chemical reactions, in the hydrodynamic limit approximation. From own results we conclude, that the chemical reaction leads to an onset of spinodal decomposition also in the case of an initial system which is completely miscible and can lead to an extreme retardation of the dynamics of the spinodal decomposition, with the probability of a general freezing of this process, which can be experimetally observed in simultaneous IPN formation.

$05.20 \mathrm{D}, 61.20 \mathrm{~L}, 64.75$
\end{abstract}

Typeset using REVTEX 
While equilibrium thermodynamics describes the overall tendency for a system to stay miscible or to realize a phase separation it is dynamics which allows one determine the time behavior of a possible phase separation, which depends on microscopic or mesoscopic parameters (e.g. self and interdiffusion coefficients, binary thermodynamic interaction parameters and the kinetic coefficients of possible underlying chemical reactions). In general two types of phase separation dynamics are expected: spinodal decomposition (SD) 1 and nucleation and growth?.

Whereas the latter remains effective for the large time regime of the phase separation, the initial regime is mainly determined by $\mathrm{SD}$, i.e. nucleation generally precedes the spinodal decomposition. Hence, there is no nucleation if a quench of spinodal decomposition is done through the critical point.

A first mean field description of the SD in metallic alloys was given by $\mathrm{B}$, and subsequently applied to the SD in binary polymer mixtures by and 0 , and is mainly determined by the influence of the complicated dynamical properties of the polymer chains on the unmixing kinetics.

In the following we investigate the SD with underlying irreversible chemical reactions, which changes both the composition ratio of the compounds and the effective (averaged) micro(meso-)scopic parameter of the physical dynamics. Typical cases are noninterfering simultaneous polymerization reactions of two monomer types (A and B, called components) with a characteristic mutually repulsive interaction, which are involved in interpenetrating polymer networks (IPNs) 6 (or semi-IPNs acteristic behavior, that with increasing time the average size of the molecules increases (in the case of a percolation-like gelation it becomes infinite for a finite-critical- time) and therefore the diffusion coefficients decreases rapidly, i.e. the dynamics of the phase separation becomes very slow and possibly produces an irreversible freezing of the SD in an stationary state. Chemical preparation techniques 8 and following evaluations using neutron scattering ${ }^{6}$ or $\mathrm{X}$-ray scattering $\mathbb{1}$ give at least a qualitative suggestion that the formation of homogeneous IPNs can be interpreted as a freezing of spinodal decomposition by irreversible 
chemical reactions, see also6. Unfortunately, quantitative, time-dependent experiments are missing up to now.

We describe the formation of IPNs (or equivalent systems) by the use of volume fractions $\varphi_{i}^{\alpha}$, where $\alpha$ determines the component (A or B), $i$ characterizes the different molecular clusters of one component. In many cases it is sufficient to use as $i$ the number of monomers of the different clusters, e.g. $\varphi_{i}^{A}$ is the volume fraction of all clusters with $i$ monomers of type A. For each compound $(\alpha, i)$ the mass balance law holds in the hydrodynamic limit

$$
\frac{\partial \varphi_{i}^{\alpha}}{\partial t}+\nabla \mathbf{J}_{i}^{\alpha}=Q_{i}^{\alpha}
$$

subject to the conservation of the currents (given by the physical dynamics) $\sum_{i, \alpha} \mathbf{J}_{i}^{\alpha}=0$, the mass of both compounds $\sum_{i} \varphi_{i}^{\alpha}=f^{\alpha}=$ constant $\left(f^{1}+f^{2}=1\right)$ and the source terms $\sum_{i} Q_{i}^{\alpha}=0$ (determined by the chemical reactions under consideration of the noninterfering reactions between A and B components). Typical (but not necessarily so) are quadratic forms in the $\varphi_{i}^{\alpha}$ for the source terms, which describe reactions of the type $[i]+[j] \rightarrow[i+j]$ (cluster-cluster reaction11, 12), i.e. $Q_{i}^{\alpha}=\frac{1}{2} \sum_{j+k=i} K_{j k}^{\alpha} \varphi_{j}^{\alpha} \varphi_{k}^{\alpha}-\varphi_{i}^{\alpha} \sum_{j} K_{i j}^{\alpha} \varphi_{j}^{\alpha}$ (for different kinetic coefficients see 1 , 14 $)$. The current of each compound $\mathbf{J}_{i}^{\alpha}$ can be described in a linear theory by the well known relation $\mathbf{J}_{i}^{\alpha}=-\Lambda_{i}^{\alpha}{ }_{\beta}^{j} \nabla \mu_{j}^{\beta}$ (note, that we use here Einstein's sum convention) with the chemical potential $\mu_{j}^{\beta}$ of the compound $(\beta, j)$ and the generalized Onsager coefficients (operators) $\Lambda$, which lead in Fourier space to the representation $\mathbf{J}_{i}^{\alpha}(\mathbf{q})=-i \mathbf{q} \Lambda_{i \beta}^{\alpha}(\mathbf{q}) \mu_{j}^{\beta}(\mathbf{q})$. Following the arguments oft, $\Lambda$ is defined by $\Lambda_{i \beta}^{\alpha j}=\delta_{\beta}^{\alpha} \delta_{i}^{j} D_{i}^{\alpha} S_{i}^{\alpha}(\mathbf{q}) \overline{\varphi_{i}^{\alpha}}$ with the diffusion coefficient $D_{i}^{\alpha}$ and the static structure factor $S_{i}^{\alpha}(\mathbf{q})$ of the compound $(\alpha, i)$, respectively $\left(\overline{\varphi_{i}^{\alpha}}\right.$ is the averaged (probably time-dependent) volume fraction of this compound). The chemical potential follows from the free energy density of a mixture

$$
F=\sum_{\alpha, n}\left[\frac{\varphi_{n}^{\alpha}}{n} \ln \varphi_{n}^{\alpha}+\frac{g_{n}^{\alpha}}{2}\left|\nabla \varphi_{n}^{\alpha}\right|^{2}\right]+\frac{1}{2} \sum_{\alpha, \beta, n, m} \chi_{\alpha \beta}^{n m} \varphi_{n}^{\alpha} \varphi_{m}^{\beta}
$$

with the Flory-Huggins parameters $\chi_{\alpha \beta}^{n m} 16$, which describe the effective binary interaction between the components $(\alpha, n)$ and $(\beta, m)$. Because this interaction is mainly determined by the monomers (and only influenced to a small degree by the molecular topology), we 
can use the more convenient simplification $\chi_{\alpha \beta}^{n m}=\chi_{\alpha \beta} \cdot g_{i}^{\alpha}$ is a characteristic length, related to the subunit length $\ell_{0}$ of the monomers by $g_{n}^{\alpha} \simeq \ell_{0} / \varphi_{n}^{\alpha}$. Hence, using (2), it follows immediately that the chemical potential $\mu_{n}^{\alpha}=\partial F / \partial \varphi_{n}^{\alpha}$. If the deviation of $\varphi_{i}^{\alpha}$ from the average $\overline{\varphi_{i}^{\alpha}}$ is sufficient small, we can use the standard approximation of a linearized theory. Note that we derive basically a mean field rate law based solely on an assumption that the error made in linearization about the instantaneous average is small. As a consequence our theory applies only to effects in the lowest order of the kinetic coefficients and the interaction parameters $\chi_{\alpha \beta}$. On the other hand, complicated polymeric systems can be described by a mean field theory with a sufficient high accuracy 15 . However, we get from (11) for the difference $\xi_{i}^{\alpha}=\varphi_{i}^{\alpha}-\overline{\varphi_{i}^{\alpha}}$ a Langevin equation

$$
\dot{\xi}_{n}^{\alpha}=-q^{2} D_{n}^{\alpha} S_{n}^{\alpha}(\mathbf{q}) \overline{\varphi_{n}^{\alpha}}\left\{\left[\frac{1}{n \overline{\varphi_{n}^{\alpha}}}+\frac{2 g q^{2}}{\overline{\varphi_{n}^{\alpha}}}\right] \xi_{n}^{\alpha}+\sum_{\beta} \chi_{\alpha \beta} \sum_{m} \xi_{m}^{\beta}\right\}+\sum_{m} R_{n m}^{\alpha} \xi_{m}^{\alpha}+\eta_{n}^{\alpha}
$$

$\left(g \simeq \ell_{0}\right)$ in which we have supplemented the right hand side by a thermodynamic random force $\eta_{n}^{\alpha}\left(R_{n m}^{\alpha}\right.$ follows from the source term by linearization, i.e. $\left.R_{n m}^{\alpha}=\partial Q_{n}^{\alpha} /\left.\partial \varphi_{m}^{\alpha}\right|_{\varphi=\bar{\varphi}}\right)$. The correlations of the stochastic forces could be a white noise but this is not necessary as long as a spectral function characterizing it exists. The kinetic equation for $\overline{\varphi_{n}^{\alpha}}$ is the 0 -th order of the linearization of (1) and satisfies $\partial / \partial t \overline{\varphi_{n}^{\alpha}}=Q_{n}^{\alpha}(\bar{\varphi})$. Thus, (3) becomes an internal time dependence, because the quantities $\overline{\varphi_{n}^{\alpha}}$ and $R_{n m}^{\alpha}$ are now time dependent. However, in the case of an effective binary repulsion between the A and B components, we can deal with one effective $\chi$-parameter, defined by $\chi_{A B}=\chi_{B A}=\chi$, whereas $\chi_{A A}=\chi_{B B}=0$ can be assumed without any restriction. Using the ansatz $\xi_{n}^{\alpha}=\overline{\varphi_{n}^{\alpha}}\left(h^{\alpha}+\Delta_{n}^{\alpha}\right)$, with the restriction $\sum_{n} \overline{\varphi_{n}^{\alpha}} \Delta_{n}^{\alpha}=0$, i.e. $h^{\alpha}=\left(f^{\alpha}\right)^{-1} \sum_{n} \xi_{n}^{\alpha}$ (note, that because of the mass conservation the sum over all $\overline{\varphi_{n}^{\alpha}}$ of one component is a time independent constant), we get from (3) after summation over all $n$ :

$$
\dot{h}^{\alpha}=\left\{\chi f^{\alpha}\left\langle W^{\alpha}(\mathbf{q})\right\rangle-\left\langle G^{\alpha}(\mathbf{q})\right\rangle\right\} h^{\alpha}-\left\langle G^{\alpha}(\mathbf{q}) \Delta^{\alpha}\right\rangle+\left\langle\eta^{\alpha}\right\rangle
$$

with the average over all molecular clusters of one component $\langle\ldots\rangle=1 / f^{\alpha} \sum_{n} \ldots \overline{\varphi_{n}^{\alpha}}$ and the functions $G_{n}^{\alpha}(\mathbf{q})=q^{2} D_{n}^{\alpha} S_{n}^{\alpha}(\mathbf{q})\left[n^{-1}+2 g q^{2}\right]$ and $W_{n}^{\alpha}(\mathbf{q})=q^{2} D_{n}^{\alpha} S_{n}^{\alpha}(\mathbf{q})$. Note, that the source 
term gives a vanishing contribution, because of mass conservation from which it follows immediately that $\sum_{n} R_{n m}^{\alpha}=\partial / / \partial \varphi_{m}^{\alpha} \sum_{n} Q_{n}^{\alpha}=0$. The total fluctuation of the composition is given by $\Delta \xi=\sum_{n} \xi_{n}^{A}-\sum_{n} \xi_{n}^{B}=h^{1} f^{1}-h^{2} f^{2}$, whereas $\sum_{\alpha, n} \varphi_{n}^{\alpha}=1$ and this leads to $h^{1} f^{1}=$ $-h^{2} f^{2}$. Hence,

$$
\frac{\partial \Delta \xi}{\partial t}+u(t, q)[\Gamma(t, q)-\chi] \Delta \xi=N(\mathbf{q}, t)+I(q, t)
$$

with $u(t, q)^{-1}=\left(2 f^{1}\left\langle W^{1}(\mathbf{q})\right\rangle\right)^{-1}+\left(2 f^{2}\left\langle W^{2}(\mathbf{q})\right\rangle\right)^{-1}$,

$N(\mathbf{q}, t)=u(t, q)\left[\left\langle\eta^{1}\right\rangle /\left\langle W^{1}(\mathbf{q})\right\rangle-\left\langle\eta^{2}\right\rangle /\left\langle W^{2}(\mathbf{q})\right\rangle\right]$ and

$I(q, t)=u(t, q)\left[\left\langle G^{2}(\mathbf{q}) \Delta^{2}\right\rangle /\left\langle W^{2}(\mathbf{q})\right\rangle-\left\langle G^{1}(\mathbf{q}) \Delta^{1}\right\rangle /\left\langle W^{1}(\mathbf{q})\right\rangle\right]$. The function

$$
\Gamma(t, q)=\frac{\left\langle G^{1}(\mathbf{q})\right\rangle}{2 f^{1}\left\langle W^{1}(\mathbf{q})\right\rangle}+\frac{\left\langle G^{2}(\mathbf{q})\right\rangle}{2 f^{2}\left\langle W^{2}(\mathbf{q})\right\rangle}
$$

can be interpreted as a (time-dependent) kinetic spinodal curve, which describes the onset of the SD. Successive approximation, using (5) and (3) leads to a perturbation like theory for the computation of the $\left\langle G^{\alpha}(\mathbf{q}) \Delta^{\alpha}\right\rangle$. However, the right hand side of (5) plays only a secondary role (especially in the case of symmetric systems (equal chemical kinetic and physical parameters $I(q, t)$ vanishes up to the order of contributions from the thermodynamic noise). Thus, (5) without the term $I(q, t)$ can used as a preaveraged equation for the SD in systems with irreversible chemical reactions. However, the main interest is in the homogeneous part of (5), which determines the Greens-function and therefore the evolution of a small fluctuation. In the case of $\Gamma(q, t)>\chi$ an initially given fluctuation decays exponentially up to the order of the thermodynamic noise, whereas for $\Gamma(q, t)<\chi$ an exponential increase of the fluctuation occurs, which heralds the SD. Because of the time dependence of $\Gamma(q, t)$, we can determine the start time $t^{\star}(q)$ of the $\mathrm{SD}$ (for the mode $q$ ) as the solution of $\Gamma\left(q, t^{\star}(q)\right)=\chi$ (In the case, that for all times $\Gamma(q, t)<\chi$ is valid, we have $t^{\star}=0$ ). Hence, the amplitude of the composition fluctuation mode $\Delta \xi(q, t)$ scales for $t>t^{\star}$ as

$$
|\Delta \xi(q, t)|^{2} \simeq \exp \left\{2 \int_{t^{\star}(q)}^{t} u(\tau, q)[\chi-\Gamma(q, \tau)] d \tau\right\}
$$

This equation allows the determination of the evolution of the composition fluctuations (for a given mode q or after integration over all modes q; this is the local composition fluctuations) 
arising as a result of the chemical kinetics and physical (molecular) dynamics. Using the usual approximation $S_{n}^{\alpha}(q)=n\left[1+\frac{1}{3}\left(R_{n}^{\alpha} q\right)^{2}\right]^{-1}\left(R_{n}^{\alpha}\right.$ is the radius of gyration of the molecules of the molecular clusters $(\alpha, n)$, which scales as $R_{n}^{\alpha} \sim n^{\sigma}$, the exponent $\sigma$ is determined by the underlying model for the structure of the molecular clusters, e.g. in the case of a percolation like growth we have $\sigma=0.395$ for dimension $d=3$ ) and $D_{n}^{\alpha}=D_{0}^{\alpha} n^{-b}$ (e.g. $b=1$ for the Rouse model $17, b=2$ for a reptation like model 18 of diffusion), for $t \rightarrow \infty$ it follows $u(t, q) \rightarrow 0$ and $\Gamma(q, t) \rightarrow 2 g q^{2}$,i.e. the exponent in (7) becomes finite as $t \rightarrow \infty$ (Clearly, this is always the case, if at a finite critical time $t_{c}$ an infinitely large cluster can be observed) or at least $|\Delta \xi(q, t)|^{2}$ no longer scales as an exponential increase $\exp (c t)$. The importance of this effect is clear: either the SD experiences a dramatic retardation of the $\mathrm{SD}$ and therefore a later beginning of the subsequent regime of nucleation and growth or the SD even freezes into a stationary state without a subsequent segregation regime. This can actually be observed e.g. in the simultaneous formation of interpenetrating networks 6 : which leads finally to an averaged domain size in the nm-scale., i.e. the domains can at best be interpreted as microphases. This freezing effect can also observed by numerical simulations19. Finally we conclude, that the influence of an irreversible chemical reaction on the onset of the evolution of the SD can be sufficiently strong, as seen by:

1. If in the beginning of the reaction no SD occurs (i.e. for small enough $\chi$-parameter), there always exists for sufficiently small $q$ a time $t^{\star}(q)$, such that for $t>t^{\star}(q)$ the SD remains effective. Thus an system which growth by irreversible chemical reactions undergoes always the SD.

2. Furthermore, the same irreversible chemical processes, which induces the SD are in competition to the SD, i.e. the existence of the irreversible, simultaneous chemical reaction on the thermodynamic separation procedure retards extremely the SD process and leads finally to a freezing of the SD, so that irreversible fixed structures are formed. A further decomposition after the retardation time scale is controlled by elastic forces, which prevent the continuation of the SD. Therefore, the final product contains mostly 
a strong pronounced elastic tension field. The effect of the chemical reactions on the coarsening exponent in (可) after the retarded SD starts can be obtained by taking into account these increasing elastic forces. Generally, it can be expected, that because of these strong forces the contributions of the retarded SD are relatively small in comparison to the SD before retardation.

Acknowledgments This work has been supported by the Deutsche Forschungsgemeinschaft, schu 934/1-3. 


\section{REFERENCES}

${ }^{1}$ J.D.Gunton, M.S.Miguel, P.S.Sahni, in Phase Transition and Critical Phenomena, ed. by C.Domb and M.S.Green (Academic, New York, London, 1983), Vol.9.

2 Nucleation, ed. by A.C.Zettlemoyer (Marcel Dekker, New York, 1969).

${ }^{3}$ J.W.Cahn, J.E.Hilliard, J.Chem.Phys.28, 258 (1958).

${ }^{4}$ K.Binder, J.Chem.Phys.79, 6387 (1983).

${ }^{5}$ P.G. de Gennes, J.Chem.Phys. 724756 (1980).

${ }^{6}$ P.Zhou, H.L.Frisch, Macromolecules, 27, 1788 (1994).

${ }^{7}$ N. Nemirovski, M. Narkis, in Interpenetrating Polymer networks, Eds. by D. Klempner, L.H. Sperling, L.A. Utracki (American Chemical Society, Washington, 1994), p.353.

${ }^{8}$ P. Zhou, Q. Xu, H.L. Frisch, Macromolecules, 27, 938 (1994).

${ }^{9}$ M. Daout, A. Brulet, P. Zhou, H.L. Frisch, J.de Physique (France) 3, 1161 (1993).

${ }^{10}$ M. Junker, I. Alig, H.L. Frisch, G. Fleischer, M. Schulz, Macromolecules, 30, 2085 (1997).

${ }^{11}$ P. Meakin, Phys.Rev.Lett 51, 1119 (1983).

12 M. Kolb, R. Botet, R. Jullien, Phys.Rev.Lett 51, 1123 (1983).

${ }^{13}$ M. Smoluchowski Phys. Zeitschrift 17, 585 (1916).

${ }^{14}$ M. Ziff, J.chem.Phys. 73, 3489 (1980).

${ }^{15}$ P.G. de Gennes, Scaling Concepts in Polymer Physics (Cornell University Press, Ithaca, 1979).

${ }^{16}$ P.J. Flory, Principles of Polymer Chemistry (Cornell University Press, Ithaca, 1986).

${ }^{17}$ P.E. Rouse, J.Chem.Phys. 21, 1272 (1953).

${ }^{18}$ M. Doi, S.F. Edwards, Theory of Polymer Dynamics (Wiley, New York, 1987). 
${ }^{19}$ M. Schulz, H.L. Frisch, J.Chem.Phys., 101, 10008 (1994). 